\title{
Next-Generation Infrastructure and Technology Issues in 5G Systems
}

\author{
K. Cengiz, Member IEEE and M. Aydemir
}

\begin{abstract}
Next-generation technologies are being tried to develop for $5 \mathrm{G}$ wireless cellular networks nowadays by many researchers. Some key technologies provide significant improvements for 5G systems in terms of huge capacity, higher data rate, signaling overhead on the network and energy-spectral efficiency. But these technologies also bring along critical issues for 5G systems. In this paper, these major problems of $5 \mathrm{G}$ networks are discussed in terms of scarcity radio frequency spectrum, interantenna synchronization, antenna deployments in cells, network and traffic management, cost and workload. Also these challenges are expressed, presented and discussed in each section to provide prior knowledge and perspective for 5G network designers and researchers.
\end{abstract}

Index Terms-5G infrastructure challenges, 5G technologies, 5G technology challenges, Massive MIMO, Spatial Modulation and IoT.

\section{INTRODUCTION}

The number of wireless network users is increasing day by day due to the world-wide web, Internet of Things (IoT), device to device (D2D) and machine to machine (M2M) communication, cloud based applications and services. People always want to have more uplink-downlink data rates, improved quality of services (QoS), better internet and mobile experience (QoE), better video streaming. As a result, these demands bring out extremely high data traffic on mobile networks. Therefore new mobile system is required to provide these demands. Researchers and developers predict that $5 \mathrm{G}$ can be a suitable candidate to answer these demands.

On the other hand, mobile communications are expected to continue their exponential growth in the upcoming years. Thus, significant modifications and enhancements should be made to existing systems to fit huge capacity increase and the demands of the mobile users. Cisco IBSG predicts there will be 50 billion devices connected to the Internet by 2020 and this corresponds to $100 \%$ increase of the number of devices in 2015 [1].

Notwithstanding, 5G heterogeneous cellular network architecture is completely a new concept from previous

Manuscript received November 21, 2017; revised January 10, 2018. Date of publication March 15, 2018.

K. Cengiz is with the Telecommunications Department, Trakya University, 22030 Edirne, Turkey.

M. Aydemir is with the Electrical-Electronics Engineering Department, Gebze Technical University, Gebze, Turkey.

E-mails: korhancengiz@ trakya.edu.tr, murataydemir94@gmail.com. Digital Object Identifier (DOI): 10.24138/jcomss.v14i1.422 generations of mobile systems because it requires a new infrastructure.

Hence, some key technologies and new ideas which are Massive MIMO, Spatial Modulation (SM), Mobile Femtocell (MFemtocell) and separating indoor-outdoor scenarios are being developed to be used in $5 \mathrm{G}$ cellular networks.

These technologies and ideas offer some innovations and improvements to traditional mobile communication systems in terms of low latency, reliability, easier handover, higher data rates for high mobility users, less signaling overhead on the network, minimizing noise and fading effects, low cost, energy efficiency, ultra-dense communications, high throughput and and spectral efficiency.

Besides, these technologies and new ideas that are examined by us in [7] bringing along new challenges that we need to resolve. This study presents open issues and challenges which should be solved and analyzed in details to constitute $5 \mathrm{G}$ architecture. This paper is extended version of conference paper [18]. The extensions to the proceeding are follows: The Massive MIMO Challenges are described and examined more comprehensive. Another new technology which is visible light communications (VLC) and its potential issues are added to the study as new subsection. With a new section, some significant issues are also presented in this extended study.

This paper is organized as follows: Section II describes the infrastructure challenges in terms of scarcity RF spectrum and network management. In Section III, popular technologies which are Heterogeneous Networks (HetNets), Massive MIMO, Spatial Modulation (SM), Distributed Antenna Systems (DAS), Machine to Machine (M2M) and Visible Light Communications (VLC) are described and some disadvantages of these technologies are analyzed. In Section IV some additional technology issues are presented and discussed. Finally, Section V concludes the paper.

\section{INFRASTRUCTURE CHALLENGES}

5G heterogeneous cellular architecture will bring along infrastructure and technology challenges. In this section, we present and discuss infrastructure challenges which are scarcity RF spectrum and network, traffic management that can cause problems while building $5 \mathrm{G}$ mobile system architecture.

\section{A. Scarcity RF Spectrum}

With the development of technologies, high computing mobile devices such as smartphones, tablets and smartwatches 
become very popular nowadays. Novel ideas and developed technologies such as Internet of Things (IoT), M2M communication and MFemtocell concept show that the demand to these developing technologies will continue day by day.

As a result, in $5 \mathrm{G}$, RF spectrum will be utilized by more mobile devices in the near future. Scarcity spectrum prevents the addition of mobile users because capacity is limited. On the other hand, intense spectrum is directly related with the total system capacity. If we want to add new wireless users in current systems, we need to increase capacity because $4 \mathrm{G}$ mobile system reached its theoretical limit.

Eq. (1) shows capacity of the channel which is defined by Claude Shannon. The total system capacity is equal to sum capacity of all sub-channels and heterogeneous network (HetNet) channels. In this formula, $B_{i}$ is the bandwidth of the ith channel, $\mathrm{N}_{\mathrm{p}}$ is the noise power, and $\mathrm{P}_{\mathrm{i}}$ denotes the signal power of the ith channel. In this point, total system capacity can be increased by three options: increasing network coverage, increasing number of sub-channels and increasing bandwidth [2].

$$
\mathrm{C}_{\text {sum }} \approx \sum_{\text {HetNetChannels }} \sum \mathrm{B}_{\mathrm{i}} \log _{2}\left(1+\mathrm{P}_{\mathrm{i}} / \mathrm{N}_{\mathrm{p}}\right)
$$

Note that increasing the capacity is not only way to solve scarcity spectrum problem. Some new technologies can be used to solve this problem. One of them can be Cognitive Radio Network (CRN) which utilizes coexistence licensed and unlicensed spectrum thus it is possible to decrease wavelength. Another solution can be mm-Wave-Visible Light Communication (VLC) as a wireless communication system instead of using RF spectrum [3]. In two technologies, carrier frequencies are higher than in traditional RF communication systems and also generally two of them use enough data rate in short range applications because data is carried by intensity of light [15].

Intense RF spectrum brings significant challenges in $4 \mathrm{G}$ mobile systems. But if Eq. (1) can be re-designed for new architecture of $5 \mathrm{G}$ via implementing the novel technologies which are shown in table 1 then higher capacity can be achieved in $\mathrm{RF}$ spectrum of $5 \mathrm{G}$.

TABLE I

HOW TO INCREASE TOTAL SYSTEM CAPACITY

\begin{tabular}{ll}
\hline \multicolumn{1}{c}{ Operations } & \multicolumn{1}{c}{ Technologies } \\
\hline Increase network coverage & Heterogeneous Networks (HetNet) \\
& Mobile Femtocell concept \\
Increase number of sub- & Massive MIMO \\
channels & Spatial Modulation (SM) \\
& Distributed Antenna Systems (DAS) \\
Increase bandwidth & Cognitive Radio Network (CRN) \\
& mm-Wave Communication \\
& Visible Light Communication (VLC) \\
\hline \hline
\end{tabular}

\section{B. Network and Traffic Management}

With a huge increase in the usage of mobile data, it becomes really hard to manage such a large network. Due to the increase in total capacity and assuming users will utilize new technologies such as MFemtocell, V2V and M2M, thus data traffic will increase significantly in $5 \mathrm{G}$ networks. Here, to manage such a large traffic and optimize data transfer between users and Internet, some new standards which are Selective IP Traffic Offloading (SIPTO), Local IP Access (LIPA) and IP Flow mobility (IFO) can be used

While managing network traffic, it will be useful to distinguish incoming and outgoing traffic. In network systems, incoming traffic is called as downlink and outgoing traffic is denoted by uplink. Asymmetric speed values of incoming and outgoing traffic make difficult to manage data traffic properly. According to the researchers, separating uplink and downlink speed will be implemented in $5 \mathrm{G}$ wireless communication systems. Therefore, two different paths will be defined for uplink and downlink. However, this approach will require additional bandwidth.

5G mobile system consists of heterogeneous multiple networks such as Cognitive Radio Network (CRN), mobile femtocell concept, large MIMO network, core network and Internet. By this reason, it is hard to manage such a large network with traditional methods. Therefore, researchers propose to design software based network management instead of using conventional methods such as routers and Layer-3 switches. In traditional methods, all managing operations are performed by using physical devices and their configuration types, thus there is always need to be used extra equipments to make network management tasks. In addition, in order to make changes to these devices, a network admin has to connect to each device and make the configuration changes manually. To add complexity to each device, user interface and syntax will makes this operation a time consuming task. All these challenges push researchers into a new idea which does not require physical devices. This new idea is called as Software Defined Networking (SDN) [12]. In parallel with the development in software defined radio (or cognitive radio), in wireless communication systems, the SDN is also improved. The main goal of SDN is to enable to make abstraction of low level network functionality into virtual services. In this technology, the network control plane is decoupled from the network data plane which significantly simplifies network management and facilitates configuration changes into the network [4]. The control plane makes decisions about the locations of the packets. Data plane physically moves the packets through the device and transmits them through the network. SDN basically proposes that the control plane should be centralized so that the configuration of the network can be maintained and changed in a highly adaptable way. With SDN, when network admins need to make change to existing configurations or regulations about data routing they do not need to connect to the multiple devices and make manual changes. The change is performed centrally and also it allows them to form and adapt the network in a highly efficient and rapid way.

\section{TeChNOLOGY CHALlENGES}

Novel technologies provide benefits and improvements on the system on the other hand each novel technology brings out some challenges which need to be solved. These challenges are unique in each technology and they need to be analyzed separately. In this section, new key technologies such as 
Massive MIMO, Spatial Modulation (SM), Distributed Antenna Systems (DAS), Mobile Femtocell, Visible Light Communication (VLC), Cognitive Radio Network (CRN) and Heterogeneous Networks (HetNets) are described and some disadvantages of these technologies are expressed.

\section{A. Antenna Deployment Problem in HetNets}

One of the important ideas of $5 \mathrm{G}$ wireless system is properly being utilized of DAS by separating indoor and outdoor user scenarios. In conventional systems which are being still used in $3 \mathrm{G}$ and $4 \mathrm{G}$ systems, the main BS is placed approximately in the center of the cells and this provides coverage for related heterogeneous network. In this way, both users in indoor or outdoor make direct connection to main BS, thus user locations are not important in the cells. This is not a problem for indoor users however it brings some disadvantages while outdoor users communicate directly with BS. For this reason, outdoor users become remote to the main BS, the signal power attenuates and this causes to penetration loss. As a result, data rate is exposed to significant damage. To solve these problems, researchers offer Distributed Antenna System with separated scenarios. In DAS, it is aspired to decrease the number of antennas at the main BS instead of placing dozens of antenna on BS. For instance, if 50 antennas are used in main BS for traditional methods, 10-15 antennas are sufficient in the new concept for main BS and the rest can be distributed in cells. Antennas which are deployed in cells have connections to the BS with fiber optics. In DAS system, at first, outdoor user communicates with the nearest antenna then the signal which comes from outdoor user that is used for establishing connection with the main BS. Therefore, this signal reaches to the main BS regardless of the user position in HetNet. This novel approach and whole HetNet structure are shown in Fig. 1. HetNet is a network which includes computers and mobile devices with various operation systems or protocols. The 5G cellular architecture should be heterogeneous with small cells, micro-macro-pico cells and relays. Fig. 1 clearly shows that $5 \mathrm{G}$ heterogeneous networks consist of different sub-networks which are proposed to be used for high mobility users [13].

The main challenge of this new technology is the placement of hundreds of antennas together with fiber connections between antennas and BS. In this situation major challenges are placing the antennas and doing this positioning into densely locations.

\section{B. Other Challenges for HetNets}

Distinct cells show different properties, for example, large cells include high-power evolved nodes (eNBs) and this causes to challenges while finding deployment opportunities for macro-eNBs. Small cells contain low-power BS or Remote Radio Heads (RRHs) and this ensures better indoor coverage. A macro-eNBs serve to macro cells and low-power BSs serve to small cells. This architecture is also shown in Fig. 1.

HetNets include small cell groups. However, HetNets are designed to comprise diverse frequency bands, macro cells in licensed spectrum (e.g., LTE), small cells in licensed or unlicensed spectrum (e.g., WiFi) and new higher frequency spectrum (e.g., mm-wave and visible light). This higher frequency spectrum can be applied to small cells in order to obtain ultra dense throughput [5].

Some other challenges for HetNets can be defined as follows:

\section{1) Inter-cell interference}

Another challenge for HetNets is inter-cell interference. This problem is directly associated with unplanned deployment of small cells because operators or service providers have no chance to determine the locations of the small cells. Additionally, devices can transmit and receive signal simultaneously, hence interference occurs in both uplink and downlink channels.

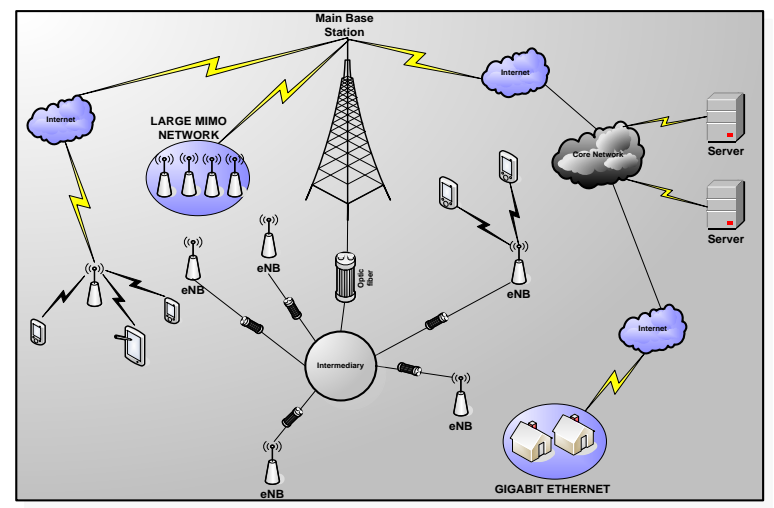

Fig 1. A 5G cellular heterogeneous network (HetNet) architecture

\section{2) Backhaul}

In 5G HetNets, macro and small cells are connected to each other via ideal or non-ideal backhauls. This causes to different levels of coordination problems across the network for mobility and interference management. If the network cooperation is increased significantly, the network capacity will be enhanced and coordination problems in the network will be solved inherently [9]. When direct access to the ideal backhaul is not available, anchor-booster architecture can be used to coordinate macro and small cells. For this architecture, the macro cell operates as an anchor BS, and it is primarily responsible for control and mobility. Furthermore, the small cell operates as a booster BS and it is mainly responsible for offloading data traffic [6]. The separation of data and control plane in anchor-booster architecture facilitates the integration of $\mathrm{WiFi}$ or future mmwave communications and VLC as booster cells within the LTE framework.

\section{3) Efficiency in medium access control (MAC) layer}

This challenge is particularly related with dense deployment of access points and user terminals which include WLANs. For this case, latency is high, handover is troublesome, and throughput is low. All these problems obligate researchers to redesign MAC layer.

\section{4) Public reliability}

Safety is the most critical challenge for all technologies. HetNet concept is robust and flexible network structure. 
Software-based management with the SDN and its flexible network architecture will prohibit physical/non-physical attacks.

\section{Massive MIMO Challenges}

Antennas are very significant components in communication systems. In existing wireless systems, number of antennas and the structures of antennas may affect the performance of cellular networks. Massive MIMO is originally envisioned from time division duplexing (TDD), but it can be applied to frequency division duplexing (FDD).

Massive MIMO can be considered as another technology candidate for $5 \mathrm{G}$ systems because it increases total system capacity and enables to increase number of sub-channels. This increment can be verified with Eq. 1. This technology includes MIMO principle for both receiver and transmitter sides. Also it allows to attain high resolution beamforming and this is very useful especially for higher frequencies and hence antenna size can be minimized significantly [11].

Massive MIMO includes significant improvements in terms of reliability, spectral efficiency and energy efficiency. In Massive MIMO systems, the transmitter or receiver modules are equipped with multiple antenna elements (e.g., ten or hundreds). Massive MIMO systems provide significant improvement on both energy efficiency and spectral efficiency. They make significant enhancement in cellular throughput. Another benefit of massive MIMO is reduced latency. Furthermore, massive MIMO systems have simplified medium access control (MAC) layer design and reduced intra-cell interference that uses simple pre-coding method.

Right along with the advantages of the massive MIMO systems, there are also important problems which are given below:

\section{1) Fast processing algorithm}

As with Massive MIMO, massive amount of data are transmitted and/or received. To interest in a huge amount of data from the RF chain, extremely fast algorithm is required in order to process these huge data.

\section{2) Extra antenna requirement}

Massive MIMO technology uses a large number of antenna arrays. Thus, this technology becomes costly in the short term because of extra antennas. Nevertheless, this effect can be ignored in the long term [14].

\section{3) Channel estimation}

Massive MIMO is originally envisioned from TDD operation. In existing systems, there is only time division duplexing process can be used for massive MIMO due to channel estimation and feedback [16]. When the number of antennas initiates to increase, channel calibration operation will become more complex than traditional MIMO systems.

\section{Inter-antenna Synchronization for Spatial Modulation}

Spatial Modulation (SM) is the low complex implementation of MIMO system. SM provides better system performance than traditional MIMO systems. The main goal of SM is to provide spectral efficiency by utilizing spatial dimension efficiently [7]
Before transmitting bits/symbols, data stream is prepared for transmission and this is called as mapping. When this operation is realized, some mapping rules should be taken into account. SM operation initiates with the mapping of data stream according to the mapping rule. Then separation of data streams into bits or symbols is realized. Divided data streams are transmitted by multiple antennas. Finally, these data streams are received with multiple antennas in the receiver side. The most specific point in SM is that there is only one antenna becomes active during all transmission periods. In this modulation technique, some part of the bit stream is selected to enable antenna, then the enabled antenna sends acknowledgment signal to another antennas to provide them to go to the sleep/standby mode collaboratively. In SM, all antennas must send acknowledgment signal in turn. Here, inter-antenna synchronization becomes very important issue. SM offers more efficient utilization of MIMO however if any error or wrong order occurs during this sequential transmission this can result in full data loss at the receiver side. Each acknowledgment signal is broadcasted and hence transmission pattern can be known by all antennas. Additionally, SM is highly related with massive MIMO therefore it uses a large number of antenna arrays. So, the mentioned challenges in above paragraphs which are channel estimation and feedback are also valid for SM.

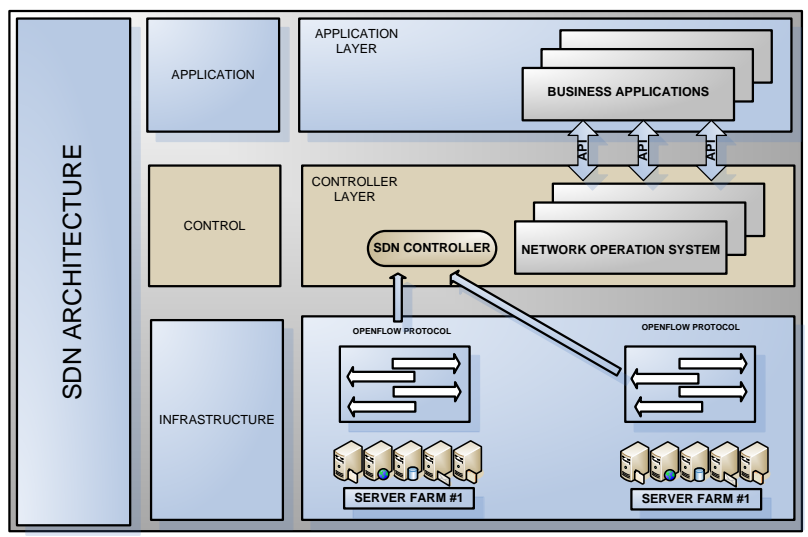

Fig 2. Software defined network (SDN) architecture

\section{E. Machine to Machine Communication Challenges}

Machine-to-machine (M2M) communication is becoming significant research field for mobile systems nowadays. Due to the developments in business areas, improvements in novel models for wireless communications and common use of intelligent life technologies such as Internet of Things (IoT) and MFemtocell concept, M2M communication becomes significant hot-topic for next generation wireless technologies. Basically, the objective of $\mathrm{M} 2 \mathrm{M}$ is to provide connection between machines and programmable smart environmental materials without human interaction to make easier daily life [10]. Nevertheless, M2M includes considerable challenges due to the usage of large number of devices with massive access for small size data. In the following sub-titles some challenges of M2M communication are expressed:

\section{1) Service requirements}

In M2M, a huge number of wireless devices are connected 
with each other. It is very hard to manage such a large wireless network. To manage this network properly, service requirements should be well-defined.

\section{2) M2M battery life modeling and life time optimization}

Considering large M2M traffic characteristics, power consumption of M2M devices have significant importance because lifetime should be prolonged in these types of networks. Life span of communicated machines is extremely important especially in health care and real-time applications. Additionally, time-to-live (TTL) and sleep mode settings should be optimized according to battery life modeling [8].

\section{3) Latency}

Latency is one of the major challenges in M2M networks, especially for the machines which work in real-time and in health industry. If this machine is thought to work as online the slightest latency can cause to fatalities.

\section{4) Massive access/traffic volume}

Compared to human-to-human $(\mathrm{H} 2 \mathrm{H})$ traffic in cellular networks, overmuch number of M2M devices cause serious challenges in radio access networks (RANs). This traffic volume uncovers signaling overhead on the network. Currently, newly designed clustering access types, M2M prioritization and management of massive access in M2M can decrease signaling overhead on RAN.

TABLE II

CHALLENGES OF TECHNOLOGIES

\begin{tabular}{ll}
\hline \multicolumn{1}{c}{ Technologies } & \multicolumn{1}{c}{ Challenges } \\
\hline Distributed Antenna System & Antenna deployment in cells \\
& $\begin{array}{l}\text { Defining usage density regions } \\
\text { Optic fiber/Indoor AP cost }\end{array}$ \\
& Inter-cell interference \\
HetNets & Backhaul \\
& Efficient medium access control \\
& Public safety \\
& Fast processing algorithm \\
Massive MIMO & Require extra antenna \\
& Channel estimation/feedback \\
& Inter-channels interference \\
Spatial Modulation & Inter-antenna synchronous \\
& Services requirements \\
M2M communication & Battery life modeling and life time opt. \\
& Security and privacy \\
& Massive access \\
& Requisite new infrastructure \\
& Management of multi-femto users \\
Mobile Femtocell & Requiste optical infrastructure \\
& Short range \\
Visible Light Communication & Implement in mobile system \\
&
\end{tabular}

5) Security and privacy

The security is a parameter that should be considered and properly implemented to every step of applications. Different M2M applications may have different privacy requirements which should be taken into account by system designers and cyber security analysts. Even if there are different kinds of applications, security standardization should be performed to cover all of them. For this case, a multi-layer architecture and authentication, authorization and accounting (3A) methods can be used. Basically, multi-layered system provides various security necessaries for communication between M2Ms. Authentication pattern offers pre-authenticated accounts to get access to the system. Authorization policy enables to authorize machines in order to accomplish various tasks. M2M and account methods keep a record of what is being done with authenticated accounts and authorized machines. This recording operation is called as logging and these logs are stored in the system database (cloud).

\section{F. Visible Light Communication (VLC) Challenges}

Visible Light Communication (VLC) is one of the data communication types which utilizes optical wireless communication schemes for short-range communications. VLC technology uses various frequency gaps between $400 \mathrm{THz}$ and $800 \mathrm{THz}$. It is based on transmitting and receiving the data in the direction of visible light principles instead of using RF signals.

Basically, VLC consists of two parts that are receiver and transmitter which are same in traditional communication systems. The VLC technology generally uses light emitted diodes (LEDs) in order to transmit the data. The receiver side uses photo-diodes (PD) or CMOS sensors. PDs can be optimum choice for VLC receivers because they convert light signals to logical symbols. In addition, PDs support Sub-carrier Pulse Position (SC-PPM) and Sub-carrier Frequency Shift Key (SCFSK) modulations. In VLC technology, data is carried by intensity of light. Signals which carry information bits have actual value and they are absolutely positive because power of signals can not be negative.

VLC provides high data rates for short-range communications. On the other hand, it is unsuccessful for long distance communications because light cannot penetrate between solid materials such as building walls. Considering the short-range novel developing communication schemes in $5 \mathrm{G}$ which are device to device (D2D) communications and machine to machine communications (M2M), VLC becomes shoo-in for these two schemes.

All above mentioned potential technologies have significant advantages in terms of various benefits. On the other hand, they can bring some disadvantages when they are decided to be used in 5G. Table II summarizes and illustrates these significant advantages and disadvantages. When a developer decides to use any of these potential technologies, developer should pay attention to the disadvantages of that system and should generate solutions for these issues. Thus, table II is presented to help developers while they are making their potential technology selection for $5 \mathrm{G}$ system.

\section{SOME AdDitional TeChNOLOGY Issues}

In this section, the potential some other technology issues which are data rate problems in cloud based applications, issues in traffic management and asymmetric data rates between uplink and downlink for $5 \mathrm{G}$ systems are discussed.

\section{A. Data Rate Issues of $5 G$ Systems in Cloud Based Applications}

Nowadays, usage of cloud based applications increase rapidly day by day. According to the forecasts, it will continue to increase. The most important advantage of cloud based 
application is to provide to access the uploaded data from anywhere at any time with various types of devices such as PC, tablet, notebook and smartphone. Through cloud applications, users carry data servers by the help of cloud services instead of making data storage in physical disks. However, users will encounter the same problem of $4 \mathrm{G}$ in $5 \mathrm{G}$ cloud based applications again. This same problem will be the limited data rates. Uplink and downlink rate will be again limited during users try to connect cloud servers and access to the data. Uplink and downlink rates of developing $5 \mathrm{G}$ systems still seem insufficient for mobile users while trying to access cloud services. Especially in uplink of 5G systems, users can not reach enough data rates to obtain satisfying connection quality. When the significant increase in user intensity for $5 \mathrm{G}$ is considered, the envisaged data rates will not meet user demands in cloud based applications.

\section{B. Issues in Traffic Management}

Because of the huge increase in the mobile data usage, it becomes really hard to manage this huge traffic in the network. Also the significant increase in total capacity causes to increase data traffic in $5 \mathrm{G}$ networks. In order to optimize data transfer between users and Internet and manage this heavy load in the networks, some new standards that are Selective IP Traffic Offloading (SIPTO), Local IP Access (LIPA) and IP Flow mobility (IFO) can be used for $5 \mathrm{G}$ networks. The usage of these technologies can solve the challenges of traffic management in $5 \mathrm{G}$ networks.

\section{Asymmetric Data Rates Between Uplink and Downlink}

According to the researchers, difference between data rates for uplink and downlink will increase when $5 \mathrm{G}$ systems are started to be used. Therefore, in $5 \mathrm{G}$ systems, two different paths are being tried to develop for uplink and downlink. However the usage of different paths for uplink and downlink will cause to be used more bandwidth by the devices. Actually many device can achieve lower energy consumption and better average cellular throughput by using different Point of Access schemes for uplink and downlink. But, each device should be managed well while dividing incoming traffic and should be aware of how it is spreading to entire network. In addition, suitable assignation of radio access network (RAN) for each device should be accomplished in new systems to solve the different data rate problems of $5 \mathrm{G}$ systems for uplink and downlink.

\section{CONCLUSION}

In this paper, we aim to present primary challenges and their solutions for $5 \mathrm{G}$ systems. Some reasonable solutions are discussed systematically in terms of several titles. Additionally, some key emerging technology issues are also discussed for $5 \mathrm{G}$ wireless systems. As a result, numerous benefits will come with $5 \mathrm{G}$, on the other hand a potential architecture of $5 \mathrm{G}$ includes some challenges that need to be solved. This paper may give a convenient background and knowledge to researchers to motivate to deliver solutions for the problems of $5 \mathrm{G}$ systems.

\section{REFERENCES}

[1] Dave Evans. "The Internet of Things How the Next Evolution of the Internet Is Changing Everything." CISCO White Paper, 2011.

Available:http://www.wwrf.ch/files/wwrf/content/files/publications/outl ook/Outlook4.pdf

[2] Wang, Cheng-Xiang, et al. "Cellular architecture and key technologies for 5G wireless communication networks." IEEE Communications Magazine 52.2 (2014): 122-130.

[3] Haas, Harald. "Visible light communication." Optical Fiber Communication Conference. Optical Society of America, 2015.

[4] Chin, Woon Hau, Zhong Fan, and Russell Haines. "Emerging technologies and research challenges for $5 \mathrm{G}$ wireless networks." IEEE Wireless Communications 21.2 (2014): 106-112.

[5] Bangerter, Boyd, et al. "Networks and devices for the 5G era." IEEE Communications Magazine 52.2 (2014): 90-96.

[6] 3GPP TR 36.932: "Scenarios and Requirements for Small Cell Enhancements for E-UTRA and E-UTRAN," v. 0.2.0

[7] Aydemir, Murat, and Korhan Cengiz. "A potential architecture and next generation technologies for 5G wireless networks." Signal Processing and Communication Application Conference (SIU), 2016 24th. IEEE, 2016.

[8] Mehmood, Yasir, et al. "Mobile M2M communication architectures, upcoming challenges, applications, and future directions." EURASIP Journal on Wireless Communications and Networking 2015.1 (2015): 1.

[9] Ge, Xiaohu, et al. "5G wireless backhaul networks: challenges and research advances." IEEE Network 28.6 (2014): 6-11.

[10] Tehrani, Mohsen Nader, Murat Uysal, and Halim Yanikomeroglu. "Device-to-device communication in 5G cellular networks: challenges, solutions, and future directions." IEEE Communications Magazine 52.5 (2014): 86-92.

[11] Di Renzo, Marco, et al. "Spatial modulation for generalized MIMO: Challenges, opportunities, and implementation." Proceedings of the IEEE 102.1 (2014): 56-103.

[12] ADVA et al., Horizon 2020 Advanced 5G Network Infrastructure for Future Internet PPP Industry Proposal (Draft Version 2.1), 2013.

[13] Damnjanovic, Aleksandar, et al. "A survey on 3GPP heterogeneous networks." IEEE Wireless Communications 18.3 (2011): 10-21.

[14] Larsson, Erik G., et al. "Massive MIMO for next generation wireless systems." IEEE Communications Magazine 52.2 (2014): 186-195.

[15] Alias, Dinu Mary. "Cognitive Radio networks: A survey." Wireless Communications, Signal Processing and Networking (WiSPNET), International Conference on. IEEE, 2016.

[16] Kuerbis, Martin, et al. "On the use of channel models and channel estimation techniques for massive MIMO systems." Signal Processing Conference (EUSIPCO), 2016 24th European. IEEE, 2016.

[17] Nie, Xiao, and Xiaobing Zhai. "M2M security threat and security mechanism research." Computer Science and Network Technology (ICCSNT), 2013 3rd International Conference on. IEEE, 2013.

[18] M. Aydemir, K. Cengiz, "Emerging Infrastructure and Technology Challenges in 5G Wireless Networks", IEEE 2nd International Multidisciplinary Conference on Computer and Energy Science (Splitech2017), Split, Crotia, July 2017.

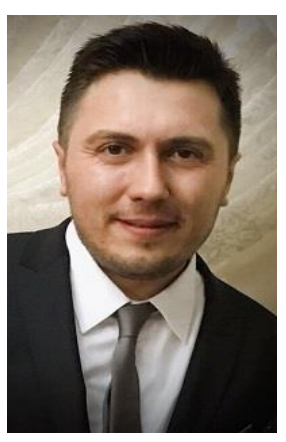

Dr. Korhan Cengiz was born in Edirne, Turkey in 1986. He received the B.S. degree in Electronics and Communication engineering from the Kocaeli University, Kocaeli, Turkey in 2008 . He received the Ph.D. degree in electronics engineering from the Kadir Has University, Istanbul, Turkey in 2016. From 2016 to 2017, he was a Lecturer Doctor and Department Head of Electronics in the ElectricalElectronics Engineering, Trakya University, Edirne, Turkey. Since Jan. 2018, he has been an Assistant Professor at the department of Telecommunications in Electrical-Electronics Engineering Trakya University, Edirne, Turkey. He is the author of more than 30 articles in IEEE Conferences and Journals and he serves as reviewer for more than 15 journals. His research interests include 5G Networks, Wireless Sensor Networks, Routing Protocols, Wireless communications, and Spatial Modulation. He is an Editor of the Turkish Journal of Electrical Engineering and Computer Sciences. Dr. Cengiz's awards and honors include the Tubitak Priority Areas Ph.D. Scholarship, ICAT 2016 Conference Best Presentation Award and Kadir Has University Ph.D. Student Scholarship. 
Murat Aydemir was born in Istanbul, Turkey in 1994. $\mathrm{He}$ received the B.S. degree in electrical-electronics engineering from the Trakya University, Edirne, Turkey in 2016. He is pursuing the M.S. degree in electronics and communication engineering at the department of Telecommunications Engineering in Gebze Technical University, Gebze, Turkey. 